\title{
OXIDATION OF DIAMOND \\ AT HIGH TEMPERATURE AND I ATM TOTAL PRESSURE WITH CONTROLLED OXYGEN FUGACITY
}

Frances A. Cull and Henry 0. A. Meyer

Department of Earth and Atmospheric Sciences, Purdue University, West Lafayette, IN 47907 U.S.A.

The presence of diamond in kimberlite is generally believed to be due to rapid ascent of a diamond-bearing magma from the mantle to the crust. The temperature of the magma plus the oxygen fugacity $\left(\mathrm{f}_{02}\right)$ and the residence time within the crust prior to intrusion at the surface all play a role in the retention or removal of diamond.

Evidence for this is perhaps illustrated by the presence of some diamonds showing resorption features, the occurrence of non-octahedral forms of diamond and the development of trigons on diamond surfaces.

It is commonly accepted that mantle and crustal fluids can be represented in part by the system $\mathrm{C}-\mathrm{H}-\mathrm{O}$ (Wyllie, 1980). Within this system the important phases are $\mathrm{CO}_{2}$ and $\mathrm{H}_{2} \mathrm{O}$ and these can be conveniently discussed as a function of the oxygen they contain i.e. f02. Evidence from coexisting iron-titanium oxides (Haggerty and Tompkins, 1983) and from a thermodynamic treatment of assemblages of olivine-orthopyroxene-ilmenite (Eggler, 1983) yield a $f_{02}$ for diamond-bearing magmas in the range bounded by QFM and MW buffers.

In the study reported herein experiments were undertaken to determine a kinetic model for the rate of transformation of diamond at high temperature $\left(900-1000^{\circ} \mathrm{C}\right.$ ) and controlled $f_{02}$ approximately to the QFM-NNO buffers (i.e. $\log f_{02} 10^{-10}$ to $10^{-14}$ ) through use of a $\mathrm{CO}_{-} \mathrm{CO}_{2}$ gas mixture, and a calcia-zirconia solid electrolyte cell. In the majority of the experiments the diamonds used were almost perfect octahedra, about 1 $\mathrm{mm}$ on edge and approximately 2 to $3 \mathrm{mg}$ in weight. Octahedrons were chosen for several reasons including the fact that the octahedron is considered to be a growth form and also that trigons develop on 111 faces. All the diamonds were clear, colorless and free of macroscopic inclusions. After weighing the diamonds were placed in a Pt capsule and suspended in the furnace at the required temperature and $f_{02}$. The capsule (t diamond) were periodically quenched, the diamond reweighed and then returned in a new $\mathrm{Pt}$ capsule to the furance. Individual diamonds were normally held at $T$ and $f_{02}$ for 12 to 48 hours between quenchings.

Examples of some results at $910^{\circ}$ and $935^{\circ} \mathrm{C}$ and $f_{02}$ of $10^{-10}$ and $10^{-12} \mathrm{~atm}$ are shown in figures 1 and 2. The rate constant, $K$, for each data set is given by the slope of the best fit line. In reality, this constant can only be called a pseudo-rate constant due to the fact that the diamonds that were used for these experiments were only of one size, and thus the possible influence of a change in surface area with degree of oxidation was not taken into account.

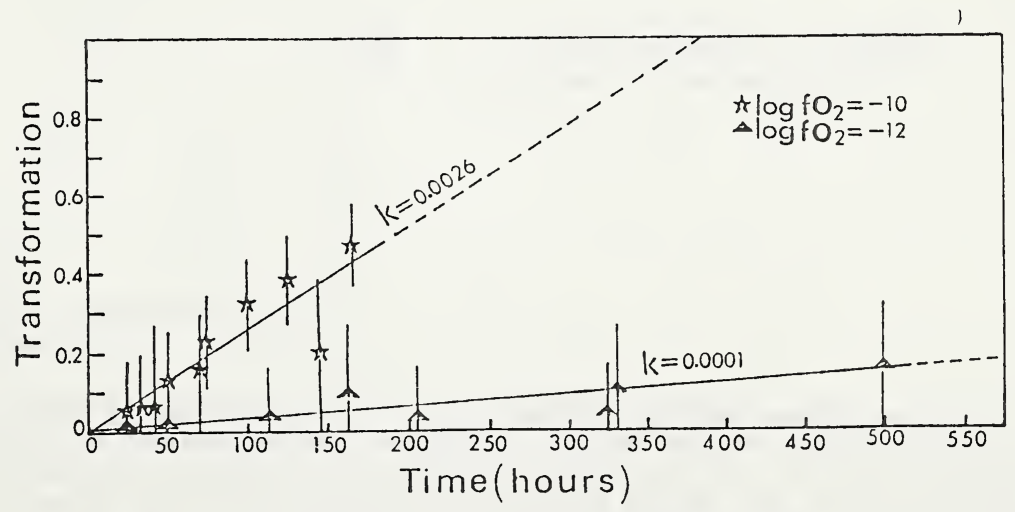

Figure 1. Temperature $=910^{\circ} \mathrm{C}$. 


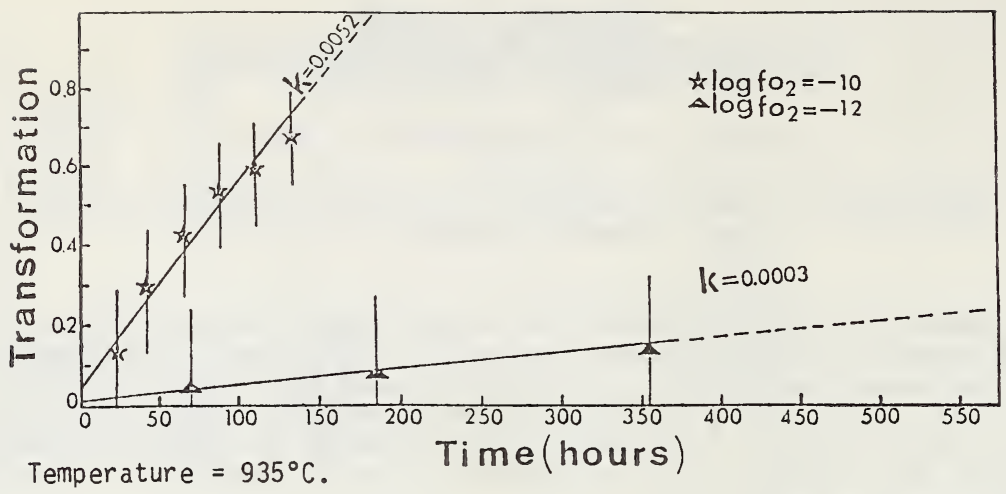

Figure 2. Temperature $=935^{\circ} \mathrm{C}$.

A feature of the surface morphology of the diamonds during and subsequent to the runs was the development of negative trigons of varying depths and sizes. It was observed that an increase in all three factors under investigation, length of time for a run, temperature and $f_{02}$ resulted in an increase in the size and frequency of the trigons that were produced. Of these three variable, f02 appeared to produce the greatest change in surface morphology. Diamonds treated at $\mathrm{f}_{02} 10^{-12}$ atm showed the greatest resemblance to diamonds as they occur in nature.

The general pattern that can be discerned from the experiments that were performed during the course of this study is that an increase in temperature at a constant fo2 produces an increase in the rate of the oxidation of diamond. Similarly, an increase in $\mathrm{f}_{02}$ at a constant temperature also resulted in an increased oxidation rate. Thus, the most rapid oxidation rates were observed for diamonds which were oxidized at higher temperatures and in the more oxidizing of the two environments. Of the two variables under investigation, examination of the rate of change of oxidation with temperature at a constant $f_{02}$ reveals that at conditions corresponding to $\log f_{02}=-10$, the rate constant increased by a factor of 9 between $910^{\circ}$ and $985^{\circ}$, while spanning a similar temperature range at $\log \mathrm{f}_{02}=-12$ produced an increase in oxidation rate by a factor of 4.2. Thus, one may deduce that an increase in $f_{02}$ resulted in an increase in oxidation rate such that the rate constant at $\log f_{02}=-10$ increased nearly twice as fast as did the rate constant at the less oxidizing conditions.

Figure 3. Arrhenius plot of data collected at $\log f_{02}=-12$.

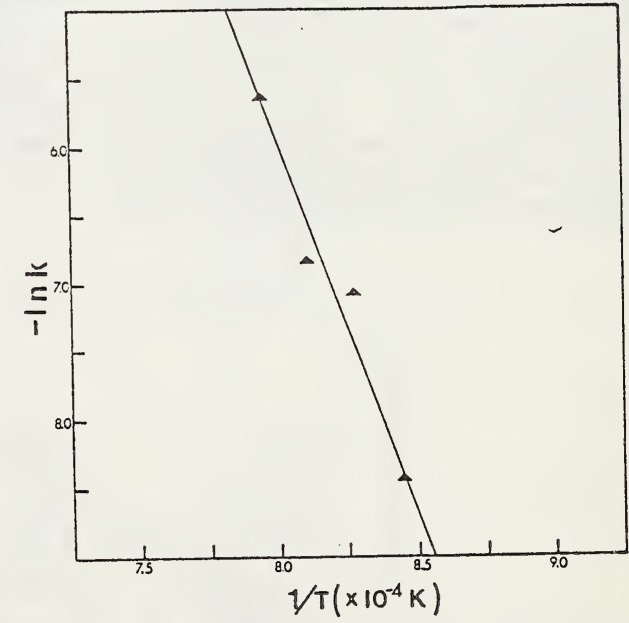

If one assumes constant surface area, then the activation energies for the diamond oxidation reaction at the two $\mathrm{f}_{02}$ conditions, $c$ an be obtained from Arrhenius plots of the data (In k vS. I/T, fig. 3). The resulting activation energies are $87.3+4 \mathrm{kcal} / \mathrm{mole}$ at $\log f_{02}=-10$ and $102.6+6 \mathrm{kcal} / \mathrm{mole}$ at $\log \mathrm{f}_{02}=-12$. It is not clear whāt the significance of these numbers are if the surface area of the diamond during oxidation is 
not taken to be constant. A possible way in which the oxidation of diamond may be viewed is as a solid state diffusion phenomenon. This is suggested by the high correlation of the data obtained with the Avrami equation $\left(\xi=1-\exp \left[-K t^{n}\right]\right.$, where $\xi=$ volume transformed, $k$ and $n$ are rate constants, and $t$ is time) for non-randomly distributed nuclei.

Based on the rate constants that can be extracted from the time vs. transformation plots, one can calculate an upper bound on how long a diamond in the size range of 2 to $3 \mathrm{mg}$ could remain at a temperature and $\mathrm{f}_{02}$ in the range studied herein before disappearing completely. This period of time ranges from 23 days at $900^{\circ} \mathrm{C}$ to 1.2 days at $1000^{\circ} \mathrm{C}$ for an atmosphere of $\log \mathrm{f}_{02}=-10$, and to 69 days at $900^{\circ} \mathrm{C}$ to 21 days at $1000^{\circ} \mathrm{C}$ for an atmosphere of $\log f_{02}=-12$. Given the rapid rate of transformation that a diamond undergoes in this temperature and $\mathrm{f}_{02} \mathrm{range}$, as is evidenced by the calculation above, one can hypothesize that a long residence of a diamond-bearing magma in a lower crustal environment of sufficiently high $T$ and $f_{02}$ would not be conducive to the preservation of micro diamond, and could cause total disappearance of such size material from the magma prior to intrusion into the upper crust.

However, it should be stressed that these values are only applicable to diamonds in the 2.0 to $3.0 \mathrm{mg}$ size range. Attempting to extrapolate from these data so as to incorporate diamonds of all sizes brings one to the quesion of the role played by surface area in the rates reported above.

Closer scrutiny of the data reveals that while the percentage of weight lost by the diamond with time at a given $T$ and $f_{02}$, increases dramatically with an increase in the degree of oxidation, the actual weight loss (in terms of $\mathrm{mg} / \mathrm{hr}$ ) remains in a limited range. Furthermore, preliminary data obtained for a diamond in the $50 \mathrm{mg}$ size range ( $25 \mathrm{X}$ the smaller ones cited above) which was subject to a temperature of $935^{\circ} \mathrm{C}$ at $\log$ $f_{02}=-10$ indicates that the weight loss per hour is essentially the same as that observed for smaller diamonds at the same conditions. This observation could be interpreted as suggesting that surface area has no major impact on the absolute rate of the oxidation of diamond, but does have an effect on the relative rate when diamonds of different sizes are compared.

\section{References:}

EGGLER, D.H. 1983. Upper mantle oxidation state: evidence from ol-opx-ilm assemblages. Geophys. Res. Letts., 10, 365-368.

HAGGERTY, S.E. and TOMPKINS, L.A. 1983. Redox state of earth's upper mantle from kimberlite ilmenites. Nature, 303, 295-300.

WYLLIE, P.J. 1980. The Origin of kimberlite. J. Geophys. Res., 85, 6902-6910. 